\title{
STATUS OF CLIC HIGH-GRADIENT STUDIES
}

\author{
H. H. Braun, S. Döbert, L. Groening, I. Wilson, W. Wuensch, CERN, Geneva, Switzerland
}

\section{Abstract}

The recent RF structure testing program carried out in the CLIC Test Facility, CTFII, is described. The main objectives of the testing program have been to gain an insight into the physical processes involved in breakdown and damage, to isolate parameters that influence breakdown and damage, and to determine gradient limits for $30 \mathrm{GHz}$ structures. The layout of CTFII in the new 'Test Stand' configuration, the instrumentation used to study breakdown and the experimental results are summarised. The new results are compared to previously published results at 11,30 and $33 \mathrm{GHz}$ produced in the context of the CLIC study.

\section{INTRODUCTION}

An important part of the CLIC study is the development of RF components and accelerating structures capable of operating at the design power of $250 \mathrm{MW}$, the loaded accelerating gradient of $150 \mathrm{MV} / \mathrm{m}$ and the pulse length of $130 \mathrm{~ns}$ [1]. Achieving such very demanding performances requires overcoming (preferably by understanding) a number of fundamental limitations including electrical breakdown and pulsed surface heating. To this end, a program of experiments to determine the physical processes involved in breakdown and a program of high-power tests of structures and components have been established.

The high-power testing program has been made at CTFII [2] which is presently the highest power $30 \mathrm{GHz}$ source available. Now reconfigured to a 'test stand' layout, it can be operated in two distinct modes. In the first it is capable of producing up to $150 \mathrm{MW}$ and up to $16 \mathrm{~ns}$ pulses of $30 \mathrm{GHz}$ RF power. In the second, single-cell standing-wave cavities can be directly powered to produce surface electric fields in excess of $400 \mathrm{MV} / \mathrm{m}$ by passing the high-current drive beam through them. All tests are made with a $5 \mathrm{~Hz}$ repetition rate. Because CTFII cannot produce the nominal CLIC power pulse, experiments and tests are specially designed to address specific breakdown issues.

During the year 2000 run, the CTFII high-gradient test program has resulted in the development of instrumentation for observing breakdown, provided insight into the physical processes involved in breakdown and has perhaps given an indication of what ultimately limits the gradient. A summary of the most important results achieved during the run are presented in this paper. Accelerating structures are presented first, followed by beam powered single-cell standing wave tests. Results are finally compared with other tests that have been made in the context of the CLIC study.

\section{ACCELERATING STRUCTURES}

The accelerating structures tested in the 2000 run were: a constant impedance structure (the cell geometry is described in [3]) with single-feed input and output couplers (tested from both ends), a constant-impedance structure with symmetrical input and output couplers and a planar structure [4]. The ratio of surface to local accelerating gradient for the constant impedance structures was 2.8 with additional factors of 1.4 in the single feed coupler and 1.12 in the symmetrical coupler respectively. Structures were baked-out in-situ to $120^{\circ} \mathrm{C}$ for two days before testing.

The structures were typically conditioned for a few times $10^{5}$ shots. The limited number of shots is due to a combination of fast conditioning, the low repetition rate of CTFII and the appearance of damage (see below). As a consequence, 'ultimate' processing may not have been done. The maximum gradients achieved after conditioning are summarised in Table 1.

Conditioning was guided by observing the currents emitted from the ends of the structures using wall current monitors. Irregular bursts of up to several hundred $\mathrm{mA}$ signalled breakdown. Pressure rises also signalled breakdown but these decreased in size as conditioning progressed. Transmitted RF pulse shortening was observed but reflected signals never exceeded $\mid \Pi=0.1$ (voltage). Missing RF energies of up to $50 \%$ were observed for breakdowns during the $16 \mathrm{~ns}$ pulses.

Table 1: Achieved accelerating gradients and corresponding surface fields.

\begin{tabular}{|c|c|c|c|}
\hline Structure & \multicolumn{3}{|c|}{$E_{a c c} \mid E_{\text {surface }}(M V / M)$} \\
\hline pulse length (ns) & 4 & 8 & 16 \\
\hline Single feed right & $133 \backslash 588$ & $90 \backslash 398$ & $59 \backslash 2$ \\
\hline Sin & 14 & $100 \backslash$ & 60 \\
\hline Plana & 70 & $0 \backslash 360$ & $50 \backslash 31$ \\
\hline Symmetrical & $120 \backslash 456$ & $95 \backslash 361$ & $70 \backslash 266$ \\
\hline
\end{tabular}

Breakdown threshold as a function of pulse length was measured for the different structures, and is summarised in Figure 1. It should be emphasised that this gives the breakdown dependence for a structure at a given conditioning state, not the dependence that a structure can be conditioned to. Although the trends are all the same, the dependencies are clearly not.

The wall current monitors were also used to measure dark current, which appeared above $95 \mathrm{MV} / \mathrm{m}$. Applying the Fowler-Nordheim analysis to measured dark current as a function of surface field gave a field enhancement factor $\beta=20$ to 30 .

A photomultiplier installed to observe visible light produced the remarkable result that breakdowns produce light pulses that last for many hundreds of ns which is far longer than the $16 \mathrm{~ns}$ RF pulses. An example of such a pulse is 


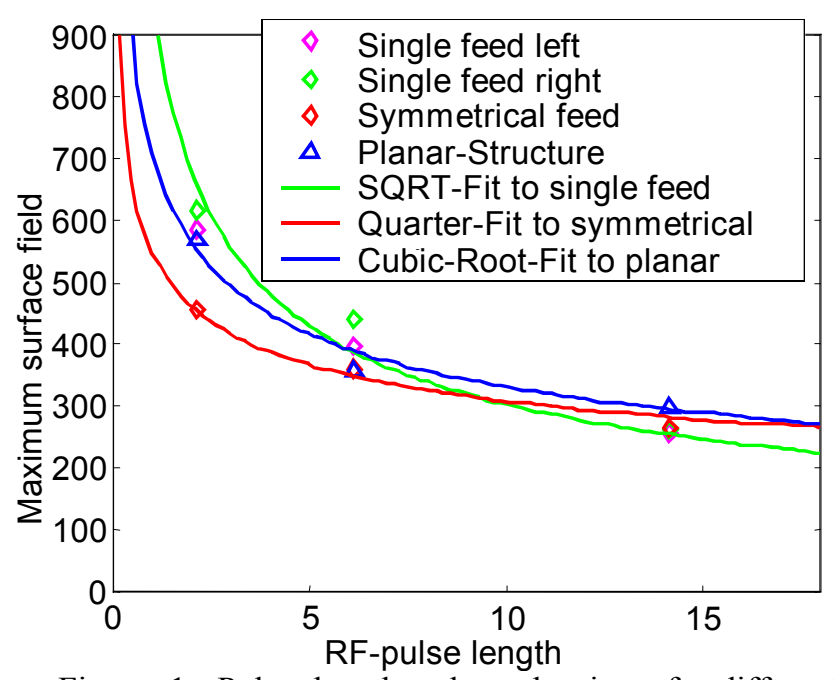

Figure 1: Pulse length dependencies of different structures.

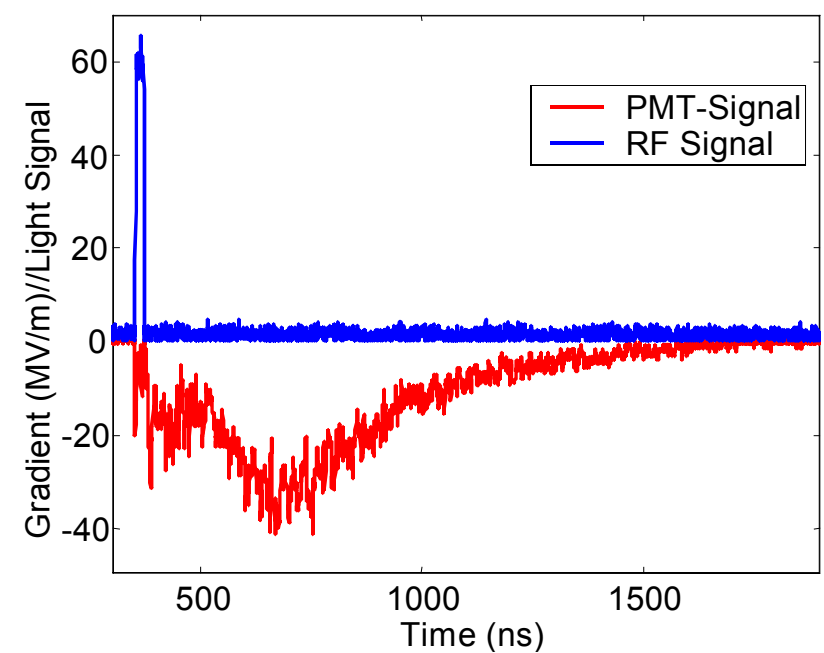

Figure 2: RF pulse and Emitted light signal.

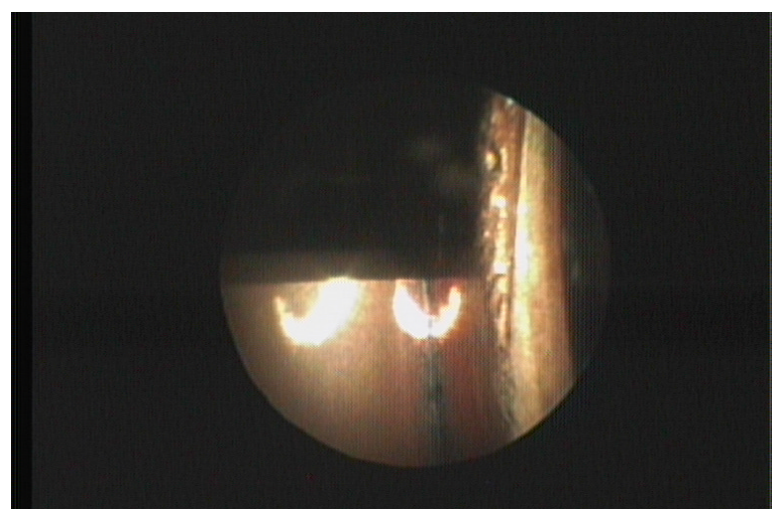

Figure 3: Damaged coupler iris, looking from the beam axis towards the coupling aperture. The section continues to the right.

shown in Figure 2. Two potential sources of long light pulses are blackbody radiation from hot copper and emission from a plasma. A measurement of the light spectrum is planned for the next run.
After the conditioning process was completed, the structures were inspected with an optical endoscope. An area of obvious damage, corresponding to a depth of removal of material of about $100 \mu \mathrm{m}$ was observed in the iris between the input coupler and the first cell. The location of the damage corresponded very closely to the field enhanced region of the couplers as calculated by HFSS [5] and shown in figures 3 and 4. Surprisingly, the damage region was delimited by a very sharp boundary.

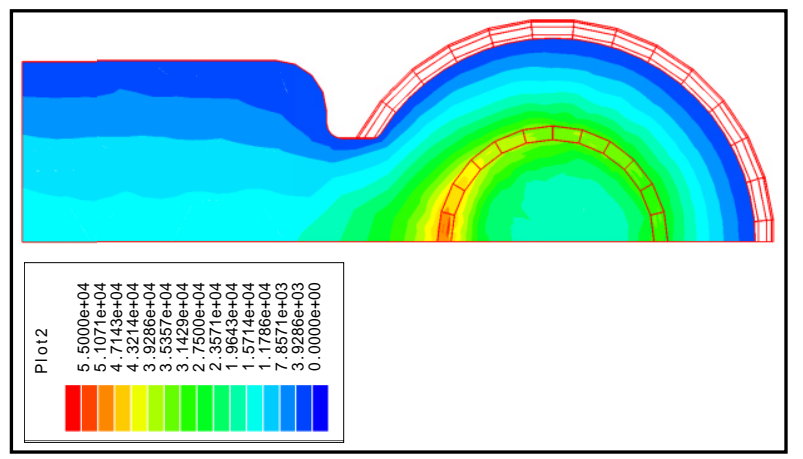

Figure 4: Simulation of the surface field distribution on the coupler iris using HFSS. The input waveguide comes from the left and the figure shows the first iris towards the disk-loaded waveguide.

\section{SINGLE-CELL STANDING WAVE CAVITIES}

Two $30 \mathrm{GHz}$ standing wave cavities were tested. The first was an optical-quality diamond-machined cavity which had already been tested in 1999 [6] and the second was a classically turned cavity. It should be stated here that previously published [6] results of field levels are incorrect (too large) by a factor $\sqrt{ } \pi$ due to a calibration error. The correct maximum surface electric field was $300 \mathrm{MV} / \mathrm{m}$.

With more beam time available than during the previous test, conditioning of the cavities was clearly observed as shown in figure 5. The maximum achieved surface fields are very close to those achieved in the travelling wave tests. The relatively simple-to-construct single-cell cavities may thus represent a valid complement to travelling-wave structures for many highgradient tests.

In an experiment whose objective was to gain insight into breakdown, the optical-quality cavity was powered while heated and cooled over a temperature range from $77 \mathrm{~K}$ (liquid nitrogen) to over $500 \mathrm{~K}$. The resonant frequency and surface resistance (computed from the measured loaded Q) behaved as expected from published values of expansion coefficient and DC conductivity. The surface resistance changed by over a factor three, as shown in figure 6. Remarkably the breakdown threshold did not change in a significant way over the entire range of temperatures, as shown in figure 7. 


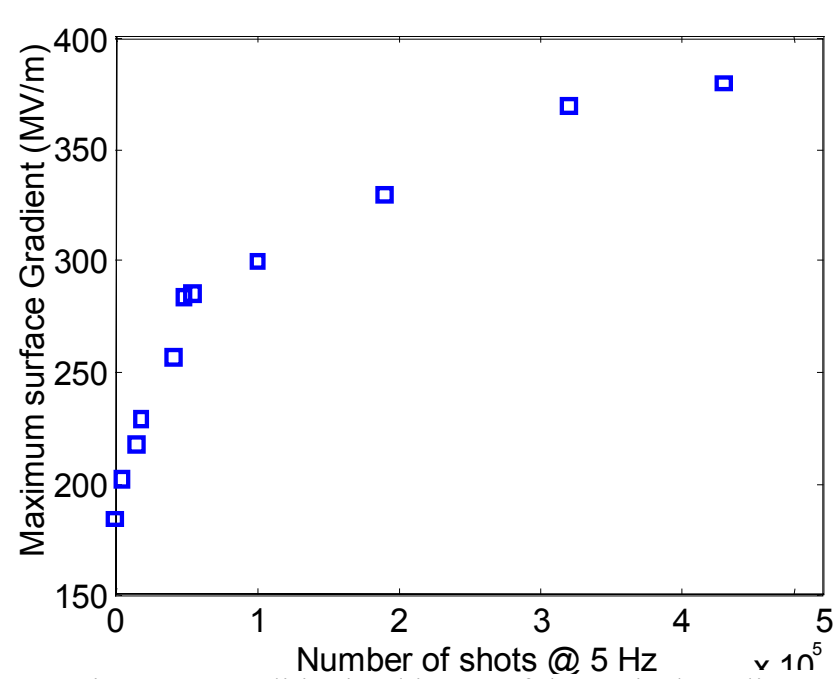

Figure 5. Conditioning history of the optical-quality $30 \mathrm{GHz}$ single-cell cavity

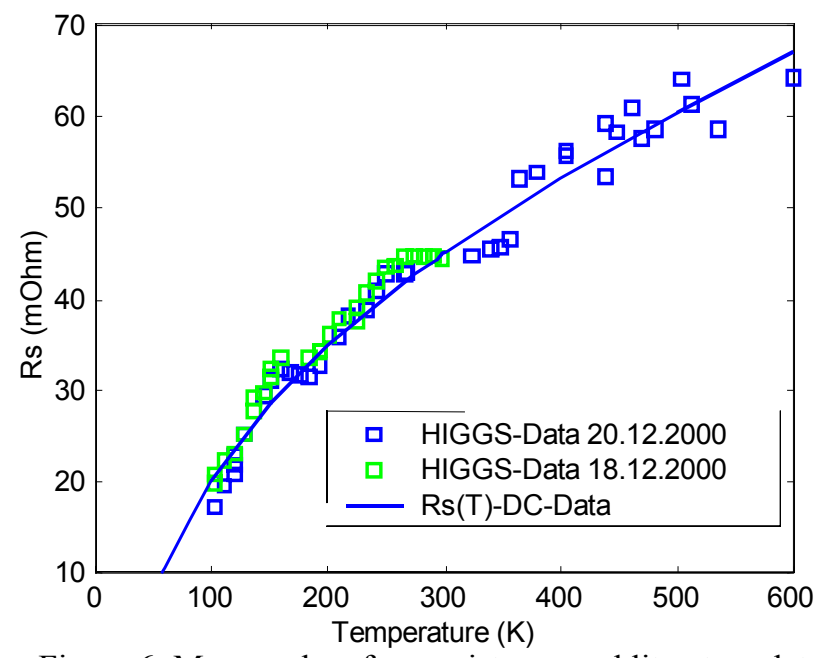

Figure 6: Measured surface resistance and literature data from DC-experiments.

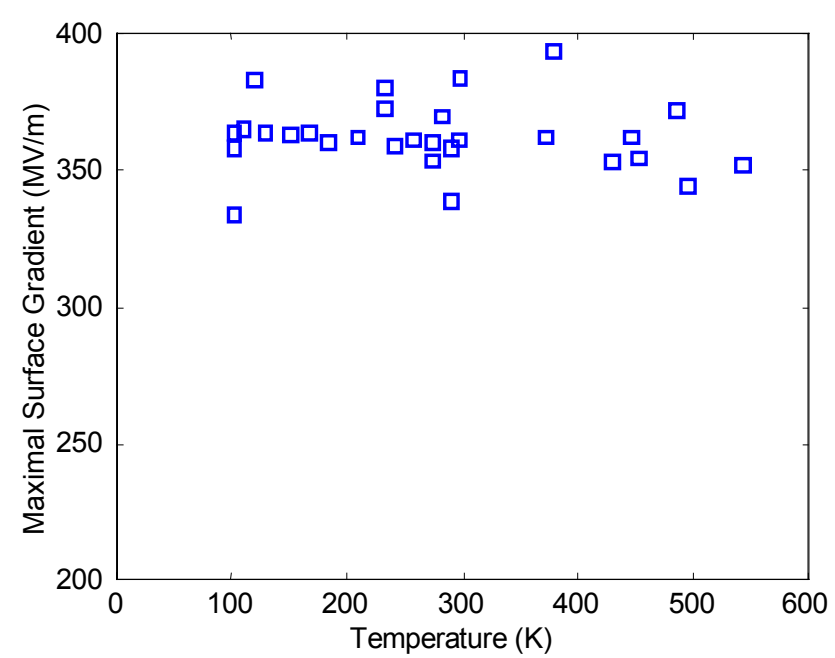

.Figure 7: Maximum surface gradient as a function of the cavity temperature.

\section{CONCLUSIONS}

The damage that has been observed in the input couplers of the travelling-wave structures is of course of serious concern. It is clear, on the other hand, that the area where the damage occurred was subject to extraordinarily high surface fields. This is a consequence of two facts. Firstly the couplers were not optimised for surface field, and the combination of asymmetry and a large and thin iris resulted in a peak surface field to accelerating gradient ratio of 4.4. Secondly the structures were tested with very short pulses. While this means that structures conditioned rather quickly, it may also mean that they were pushed beyond acceptable values of surface field for copper surfaces. The values of field enhancement of 20 to 30 and a peak surface field in the $300-400 \mathrm{MV} / \mathrm{m}$ range implies local field values of the order of $10 \mathrm{GV} / \mathrm{m}$. According to Fowler-Norheim theory, this is the value where copper is directly melted by field emitted currents. One of the paths which will be pursued to improve the achievable accelerating gradient will be to improve the surface field to accelerating gradient ratio. Another will be to investigate the potential of a material with a higher melting point and erosion resistance such as tungsten.

If the results of the CLIC X-band structure tests $[7,8]$ and the $33 \mathrm{GHz}$ test [9] are considered with the $30 \mathrm{GHz}$ data, a striking feature emerges. Despite widely varying test conditions (11 to $33 \mathrm{GHz}$, standing wave, travelling wave with $\mathrm{a} / \lambda$ from 0.11 to $0.20,500^{\circ} \mathrm{C}$ temperature range, pulse lengths from 4 to $250 \mathrm{~ns}$ ), the maximum peak surface fields achieved have consistently fallen in the 300 to $400 \mathrm{MV} / \mathrm{m}$ range. An aspect of the future experimental program will be to investigate if and how this surface field represents a fundamental limit.

\section{REFERENCES}

[1] CLIC Study Team, "A $3 \mathrm{TeV} \mathrm{e}^{+} \mathrm{e}^{-}$Linear Collider Based on CLIC Technology," CERN 2000-008, 2000.

[2] H.Braun, "Achievements and Future Plans of CLIC Test Facilities," HEACC2001, Tsuukba, March 2001.

[3] I.Wilson, W.Wuensch, "Revised Parameters for the CLIC Main Linac Accelerating Structure," CLIC Note 145, 1991.

[4] R.Merte, Thesis, Tech. Univ. Berlin, D83, 2000.

[5] I.Syratchev, private communication.

[6] W.Wuensch, W.Wilson, H.Braun, M.Luong, EPAC2000, Vienna, August 2000.

[7] T.Higo, T.Taniuchi, M.Yamamoto, J.Odagiri, S.Tokoumoto, H.Mizuno, K.Takata, I.Wilson, W.Wuensch, "High Gradient Performance of X-Band Accelerating Sections for Linear Colliders," Particle Accelerators, Vol. 48, pp. 43-59, 1994.

[8] J.Wang, G.Loew, R.Loewen, R.Ruth, A.Vleiks, I.Wilson, W.Wuensch, "LSAC/CERN High Gradient Tests of an XBand Accelerating Section," PAC95, Dallas, May 1995.

[9] P.Volfbeyn, I.Mastovsky, G.Bekefi, I.Wilson, W.Wuensch, "Experimental studies of a CERN-CLIC $32.98 \mathrm{GHz}$ high gradient accelerating structure driven by the MIT free electron laser amplifier," PAC95, Dallas, May 1995. 